\title{
PRÁCTICAS TECNOLÓGICAS MOVILIZADAS LOCALMENTE EN CONTEXTOS GLOBALES
}

\author{
Technological Practices locally mobilized in global contexts
}

Glória Baigorrotegui

\section{Resumen}

A partir de los aportes de la tradición Ciencia, Tecnología y Sociedad (CTS) y los estudios contemporáneos de las movilizaciones ciudadanas ante riesgos y proyectos tecnológicos específicos, en este trabajo renuevo la necesidad de diseñar contraproyectos tecnológicos y discuto la pertinencia de concebir prácticas tecnológicas contrahegemónicas en la escena global. Para esto primero aclararé $(0$.$) desde qué perspectiva la tecnología ha sido$ concebida como práctica social, seguidamente expondré (1.) las nociones de globalización y contrapoder. A partir de ambos acápites ubicaré el problema de (2.) la configuración de prácticas tecnológicas movilizadas. Finalmente (3.) concluiré que la interconexión de ambos fenómenos propone desafíos conceptuales y oportunidades vivenciales para las personas, grupos y movimientos de resistencia y movilización. Primero, porque interconecta las teorías de los nuevos movimientos sociales con las teorías constructivistas de la tecnología, y segundo, porque presenta la posibilidad de concretar iniciativas tecnológicas afines a demandas contrahegemónicas.

Palabras-clave: Patrones alternativos en ciencia e industria. Prácticas tecnológicas contrahegemónicas. Globalización.

\begin{abstract}
From the contributions of Science, Technology and Society (CTS) tradition and the contemporary studies of citizen mobilization in front of risks and specific technology projects, in this paper I renew the necessity to design counterprojects and I discuss the relevance to manage counterhegemonic technological practices in global scene.
\end{abstract}

For this, first I propose (0.) from which perspective the technology has been conceived as a social practice, then I discuss (1.) notions of globalisation and opposition. From both headings I situate the problem of (2.) Design of mobilized technological practices. Finally (3.) I conclude that the interconnections of these phenomena suggests conceptual challenges and experiential opportunities for individuals, groups and movements of resistance and mobilization. First, it interconnects the new social movement approaches with constructivist theories of technology, and second, because it presents the possibility to shape technology initiatives related to 
counterhegemonic demands.

Keywords: Alternative pathways in Science and Industry. Counterhegemonic technological practices. globalisation.

\section{La tecnología entendida como práctica social}

Las críticas, antagonismos y conflictos relacionados con la práctica tecnológica se han alimentado de una concepción de la tecnología y su materialidad entendida como algo determinado, que es históricamente irremediable e ineludible en la vida de las personas. En la actualidad, la tecnología como práctica se comprende desde una perspectiva distinta porque se la reconoce intrínseca en aquellos campos en donde se libran las confrontaciones y conflictos, claves para su constitución.

Un debate en torno a la tecnología, distanciado de posturas trascendentales, es aquel que discute los aspectos de su condición material, su invulnerabilidad en la manufactura (URE, 1835; KAPP, 1978), su relación con la praxis (BUNGE, 1976) y su creciente omnipresencia en el trabajo y en la vida cotidiana. Estas inquietudes académicas e intelectuales concurrieron con las movilizaciones de grupos sociales preocupados e inquietos ante las intervenciones tecnológicas en las sociedades occidentales, lo que, en conjunto, abrió vías de interés social en torno a la tecnología.

A mediados del siglo pasado, a tenor del surgimiento de una perspectiva crítica de la tecnología, enmarcada en las precursoras contribuciones de Marcuse sobre el papel de la tecnocracia en la sociedad de consumo (MARCUSE, 1964), movilizada por grupos pacifistas y antinucleares, se consolidó la necesidad de integrar a diferentes disciplinas en la reflexión sobre la ciencia y la tecnología en la sociedad. Fue en esta época cuando los Estudios Sociales de la Ciencia y la Tecnología (CTS) se hicieron más visibles y cohesionados por el propósito de destronar la imagen predominantemente determinista de la tecnología. A partir de estas emergencias se fue haciendo cada vez menos comprensible la ceguera que algunos científicos y tecnólogos parecían padecer frente a humanistas y sociólogos, y viceversa. 
Mientras tenía lugar esta polémica entre las disciplinas y sus competencias, intelectuales preocupados; unos por la ciencia, otros por la tecnología decidieron dar por terminada esta "guerra entre las dos culturas" (SNOW, 1959) y se concentraron en problematizar la noción de práctica. Los primeros (BARNES et al., 1996; BLOOR, 1976; COLLINS, 1981) concluyeron que en la construcción del conocimiento científico, hechos y valores; naturaleza y cultura cooperaban tan cercanamente que suponerlos separados para explicar la conformación de conocimiento científico resultaba incorrecto; los segundos (PINCH y BIJKER, 1984; LATOUR, 1992; CALLON, 1987; LAW, 1987a, 1987b; HUGHES, 1983), en cambio, centrados en la construcción de artefactos y sistemas tecnológicos determinaron que distanciar explicativamente artefactos de humanos o factores materiales de factores sociales era un obstáculo que debía salvarse, si el propósito por comprender la dimensión social y política de la tecnología era mantenido. Un aporte destacado en esta línea de comprensión fue el entregado por Langdon Winner (1977), quien criticó la idea de que los artefactos y los sistemas tecnológicos fuesen entidades “inherentemente políticas". Con esto Winner cuestionó toda afirmación que presentase a la tecnología como resultado del ejercicio de una lógica interna, la cual - sin influencia social alguna - fuese capaz de dar forma a la sociedad, haciéndola dependiente de sus patrones pre-establecidos. En este sentido, Winner aclaró que:

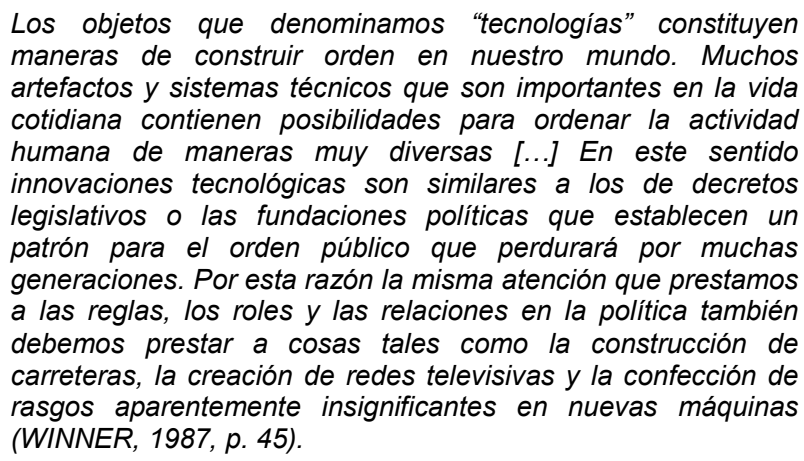
artefactos y sistemas técnicos que son importantes en la vida cotidiana contienen posibilidades para ordenar la actividad humana de maneras muy diversas [...] En este sentido innovaciones tecnológicas son similares a los de decretos legislativos o las fundaciones políticas que establecen un patrón para el orden público que perdurará por muchas generaciones. Por esta razón la misma atención que prestamos a las reglas, los roles y las relaciones en la política también debemos prestar a cosas tales como la construcción de carreteras, la creación de redes televisivas y la confección de rasgos aparentemente insignificantes en nuevas máquinas (WINNER, 1987, p. 45).

El autor con esta idea precisó que los cambios sociales, políticos y humanos deseados no podían desligarse del propio diseño, implementación 
y gestión tecnológica. Fue así como los discursos, debates y propuestas sobre el papel de la tecnología en la sociedad fueron acompañados de máquinas y sistemas diseñados, fabricados, implementados y gestionados bajo presupuestos y propósitos políticos contrahegemónicos.

En resumen, la tecnología como práctica presenta una opción para comprender las relaciones entre artefactos, conocimientos y prácticas cercanamente imbricadas desde una perspectiva crítica. Primero, propone despertar del "sonambulismo tecnológico" [II] para; por un lado, alzar la voz y decir "sí" o "no" a determinados disposiciones tecnológicas, y por otro lado, presionar con tal de participar en el diseño, o, por el contrario, para realizar acciones contenciosas y simbólicas con el fin de posicionar contradiseños en escenas públicas y políticas determinadas. Esta última opción propone oportunidades interesantes en los términos de movimientos sociales transnacionales contrahegemónicos.

\section{Globalización y contrapoder}

El fenómeno de la globalización lo ha presentado Ulrich Beck (2004) en términos de un futuro abierto bajo el marco de una Gran Política, de una Gran Transformación. Para Beck esta especie de metajuego de la política mundial desafía a las concepciones acerca de la política nacional abiertamente. Se trataría de reconocer en las formas de poder globales, sus capacidades para difuminar límites y desterritorializar a los países y, junto con ello, lograr tornar los pilares del poder estatal en objeto de estrategias de poder políticas y económicas en un estrato mundial. Esta transformación que él denomina de "segundo orden", quebranta la realidad nacional porque es la globalización y no "el Estado" quien define y transforma las arenas de la actuación colectiva" (BECK, 2004, p. 26).

Es así como las consecuencias originadas por determinadas prácticas - llevadas a cabo por organizaciones, como empresas privadas transnacionales -, se transforman en las responsables de subvertir los límites trazados por instituciones formales y oficiales. Este modo de metapolítica, Beck lo describe como una irrupción en el seno de las reglas y 
los acuerdos de convivencia previos, ante la cual el autor propone: reaccionar con tal de no quedar fuera de juego y no resultar alienado por la acción de este metapoder. Dentro de su propuesta denominada "teoría crítica con intención cosmopolita" me ceñiré en particular a la que trata las estrategias de los movimientos de la sociedad civil, porque es aquí donde se ubica mi interés por discutir y proponer vías para la configuración de prácticas tecnológicas movilizadas.

Beck, ampliando la noción de dominio de Weber, propone un tipo de "dominio" denominado "dominio translegal", al cual le asigna una forma de poder que es legal e ilegítima. A partir de aquí Beck supone que a mayor poder transnacional, menor será la legitimización democrática y, con ello, más escuálidas serán las formas de institucionalización como vías de dominio legítimo. Las fuentes de este vacío de legitimación, Beck lo sustenta en: i) el dominio translegal de la economía mundial, ii) la privatización de las funciones del Estado y su emigración a la economía mundial, iii) la transnacionalización de los Estados y sus consecuentes estrategias y formas interestatales de cooperación; y iv) los regímenes internacionales de gobernanza sin gobierno.

Dado este panorama en el cual gana terreno el tipo de "dominio translegal”, Beck muestra a las agencias que se verán beneficiadas por él. Se trata de "movimientos reivindicativos de la sociedad civil global", los que actúan como abogados creadores y jueces de valores y normas globales. En este sentido,

\footnotetext{
Crean y aguzan esta conciencia de valores (cotidiana, local y global a la vez) poniendo en escena e inflamando el horror público, la indignación del público mundial por las violaciones espectaculares de las normas; y lo consiguen de la mano de casos particulares, ya sean escándalos medioambientales de los que culpabilizan a algún consorcio pillado in fraganti, ya sean biografías de torturados con las que sacuden la conciencia (Gewissen) mundial" (BECK, 2004, p. 315-316).
}

Claro que estos movimientos tienen algunos problemas añadidos para Beck: no tienen un enemigo claro, ni tampoco cuentan con "un" lenguaje cosmopolita del conflicto, sino una "torre de babel" de lenguajes. Frente a estos problemas Beck sugiere a estos movimientos autonombrados -no 
elegidos con el voto - que distingan las posibilidades del "capital de legitimación" y, a partir de éste definan sus estrategias, las cuales propone sean tres: i) la dramaturgia de los riesgos, ii) democratización, y iii) cosmopolitización.

Beck asume que una fuente de poder no tradicional es aquella basada en la "legitimación", proveniente de una especie de virtuosismo para generar redes de cooperación internacional con tal de que se hagan visibles y se formen alianzas en temas específicos en contextos de no soberanía [III]. El desafío propuesto por Beck es aquel que propone aumentar el poder de la legitimación para lograr presionar a unos Estados mediante la alianza con otros Estados y/o consorcios, en temas medioambientales y de Derechos Humanos, forjando así alianzas de todo tipo y, por último, haciendo veraces los hechos, escenificándolos.

Aclararé que la precisión de Beck sobre la inconvertibilidad del "capital de legitimación" en "capital económico" es exigua. Las acciones que este autor propone que realicen los consumidores se centran en que ejerzan su potencial de negación. De este modo solicita que éstos nieguen su compra, actuando en su condición de no miembros, al no ser contratados por las empresas y los consorcios. Sin embargo, las acciones propuestas por Beck si bien pueden ser capaces de presionar las tendencias del mercado de proveedores hacia las preferencias de los consumidores, también es cierto que estas acciones no se libran de ser convertidas económicamente en círculos viciosos que nuevamente desfavorezcan a los consumidores [IV]. La construcción y escenificación de la información pública es una estrategia bien conocida por los departamentos de marketing y ventas. Aunque las escenificaciones de los consumidores logran transformar tendencias de compra particulares, asimismo, éstas provocan - como contraparte - ofertas renovadas de consumo, las cuales tienen el propósito de lograr la continuidad del consumidor, y con ello, trabajan en mantener la legitimidad de las empresas. Por lo tanto, esta situación no permite asegurar que ambos capitales sean completamente excluyentes, aunque sí relacionados de forma controvertida. Esta falta de evidencia en Beck rebate su supuesto que propone a los movimientos reivindicativos de la sociedad civil bebiendo 
exclusivamente de la fuente del capital de legitimación no convertible a otros tipos de capital.

En este entendido concuerdo con Appadurai (2001) en que el énfasis de la crisis de los Estados-nación se reconoce más bien en la "redundancia" y no en la "legitimidad". La "redundancia", para este autor, se traduce en el aumento de la posibilidad de conectar distintos procesos que otros han identificado. Además, este horizonte posibilista no es individual sino que surge de dinámicas entre multiples actores, y por tanto, permite que determinados procesos intencionales desde los más desfavorecidos logren ser promovidos a partir de diferentes Estados-naciones y regiones en niveles de gobernanza dispares. Con este enfoque en la desterritorialización, por sobre la deslegitimización, los activistas sin fronteras contarían con formas de poder y estrategias [v] más plurales que las expuestas por Beck; menos dependientes de la exclusiva escenificación de la información y la evidencia bajo marcos de interpretación como los de la dignidad humana y los derechos humanos. Dicho esto, no descarto las estrategias presentadas por Beck porque mantienen la alarma, por ejemplo, en torno a la correlación entre el vacío de legitimación, el crecimiento explosivo de ONGs y el fortalecimiento del discurso de los Derechos Humanos legitimado.

Si consideramos entonces el problema de "la redundancia" por sobre el vacío de la "legitimación" de las demandas desde los Estados-nación, se exhibe un área medular en los términos de oportunidades en la globalización, y aquí nuevamente retorno a Beck:

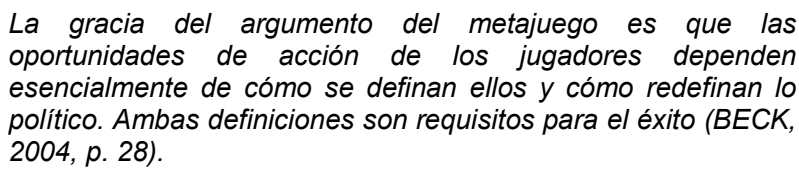

De este modo, la redefinición de la propia identidad en el metajuego es una acción reflexiva (GIDDENS et al., 1997; BOURDIEU y WACQUART, 2005). En este sentido, los resultados se asumen ambiguos aunque siempre provenientes de esta reflexión. Es en este punto crucial donde se ubica el 
planteamiento de la contrahegemonía.

La hegemonía, en su versión gramsciana, describe los procesos mediante los cuales las clases dirigentes soportan la creación y difusión de un sistema general de valores e ideas filtrándolos en la mayoría de las instituciones de la sociedad. Para Santos y Rodríguez, las ideologías, instituciones e intereses son hegemónicos porque se ven como producto del sentido común y, por el contrario, lo contrahegemónico siempre está en peligro de extinción producto de la cooptación y la desaparición (SANTOS y RODRÍGUEZ, 2007, p. 21). Así la labor de erosionar este sentido común y el sustento valorativo de instituciones del tipo hegemónico sobrepasa un trabajo de deconstrucción discursiva y simbólica, porque requiere que determinadas acciones subalternas se hagan visibles, debido a que luchan por lograr interpretaciones, prácticas y sentidos comunes renovados.

Un ejemplo de la no negación de la "comunidad de víctimas" (DUSSEL, 1998) que luego reflexiona sobre su situación y la transforma en una "política del reconocimiento desde abajo" (TAYLOR, 1997) es entregado por Arjun Appadurai (2001). Allí este autor muestra cómo la asociación entre dos grupos hindúes a favor de la lucha por el logro de viviendas con facilidades sanitarias mínimas para los ocupantes de los suburbios de Mumbai concretaron campañas de desobediencia civil y de políticas innovadoras. Un ejemplo lo entregan las iniciativas que estas agrupaciones denominaron los "festivales de aseos" en varias ciudades de la India. Estos festivales resituaron el acto privado de humillación y sufrimiento de los hindúes marginados en una escena de innovación técnica, celebración colectiva, carnaval festivalero frente a oficiales del Estado, el Bando Mundial y la clase media en general. El "festival de los aseos" no sólo inauguró modelos de aseos, sino que inauguró aseos públicos funcionando, diseñados por y para los pobres, integrando un complejo sistema de pago colectivo y mantención en condiciones higiénicas óptimas. En esta metáfora que Appadurai denominó "la política de la mierda" hubo un acto de reflexión que se volvió a sí mismo, con lo que la humillación y la victimización fueron transformadas en ejercicios de iniciativas tecnológicas y autodignificación (APPADURAI, 
2001, p. 37). Claro que este giro reflexivo no fue nada fácil debido a que lograron que los grupos más recalcitrantes se integraran en el proceso que transformó la denegación en subjetivización. Tal como expresa el mismo Appadurai:

\begin{abstract}
La "política de la mierda" - como Gandhi mostró en su propio esfuerzo por liberar a las castas más bajas, a quienes él llamó Harijans gracias a su tarea de acarrear los excrementos de las castas-altas - presenta un nodo en donde se encuentran las preocupaciones del cuerpo humano, la dignidad y la tecnología, un nexo mediante el cual los pobres son ahora redefinidos con la ayuda de movimientos como el de la Alianza (APPADURAI, 2002, p. 37 [traducción personal]).
\end{abstract}

Destaco hasta aquí que es el "vacío de legitimidad" que van construyendo los Estados y Empresas lo que favorece, en este caso, el despertar o el hastío ante las demandas locales persistentemente olvidadas por las instituciones. En este sentido, no sólo el "capital de legitimación" es un recurso importante para la reivindicación, sino también "la redundancia" de las conexiones y construcciones locales - la mayoría de ellas enganchadas en redes mundiales -, los que permiten ampliar el repertorio de confrontaciones en favor de las identidades y personas amenazadas, víctimas, marginadas, preocupadas, etc. Así Appadurai sugiere que el espacio de posibilidades transfronterizo para la lucha contrahegemónica se constituye a partir de un ejercicio de reflexión de los grupos resistentes centrado, no sólo en la conformidad de sus demandas, sino también en la persistencia y materialidad de sus acciones dentro de las condiciones globales de intercambio.

\title{
2. Prácticas tecnológicas movilizadas
}

David Hess (2007) propone destacar el concepto de "vías alternativas" en la configuración de la ciencia y la tecnología. Bajo esta modalidad Hess propone hacer visible aquellos intersticios de las categorías sociales donde se ubican aquellas personas que interpretan como desarraigo la actividad de consorcios económicos y políticas distantes a sus experiencias en 
comunidad. Este entramado de poder es percibido como agencias irresponsables frente a las necesidades de las personas de contar con instituciones democráticas, desarrollar trabajos dignos, expresar materialmente sus culturas, y afianzar sus relaciones personales. Asimismo, las "vías alternativas" representan la preocupación por articular un mundo distinto a la globalización corporativa, en la cual ellas mismas se perciben cogidas junto a sus bien intencionados planes, tecnologías, conocimientos, organizaciones, y productos, los que con frecuencia se ven amenazados cuando las instituciones económicas y políticas más importantes reconfiguran sus alternativas. Es para Hess la consideración renovada de una especie de dialéctica entre la oposición y el compromiso, o, entre la incorporación y la transformación.

Las acciones movilizadas frente a tecnologías determinadas tienen el objeto de afectar en los procesos de innovación industrial y cambiar el curso de la historia en un campo tecnológico.

Hess destaca que las principales estrategias en este campo son llevadas adelante por los denominados movimientos de oposición industrial. Como movimientos éstos no obedecen a las categorías propias de los movimientos sociales, son más bien organizaciones de alcance modesto, propio de las redes de activismo en campañas particulares. Las asociaciones profesionales, empresariales con objetivos de transformación de largo alcance se conectan con estos movimientos [VI] y, a su vez, los movimientos pueden reclutar a expertos en sus filas con el fin de proveerse de una contraexperticia.

Estos movimientos trabajan en torno al objetivo de parar una tecnología particular y, a menudo, también enfocan su denuncia hacia el sistema de relaciones más amplio en el cual ésta se encuentra inmersa. Hess menciona como ejemplos de estas relaciones a las prácticas de producción el entramado industrial y de consumo, la contaminación generada por ellos, la responsabilidad social de las empresas, las agendas de investigación discutidas entre empresas y universidades, la gobernabilidad del Estado y su definición de políticas industriales, entre otros. 
La posibilidad de configurar la tecnología por parte de movimientos sociales, particularmente los medioambientalistas, también ha sido esbozada preliminarmente por Adrian Smith (2005). Para este autor existirían dos modos de configurar las prácticas tecnológicas: i) re-enmarcar, re-frame, determinados aspectos tecnológicos y/o ii) negociar la participación de otros actores en las líneas tecnológicas. En ambas formas, se observa la confluencia de los enfoques acerca de la construcción de artefacto, por un lado, y el origen de movilizaciones sociales, por otro. En detalle Smith expone ambas modalidades así:

Modo I: Re-enmarcar las tecnologías, los movimientos medioambientalistas actuarían en campañas en las cuales pueden lograr ampliar los criterios de valoración y los estándares tecnológicos, además de alterar las posiciones relativas de diferentes opciones tecnológicas, por ejemplo, las renovables por sobre las fósiles.

Las acciones directas, como la organización de "boicots" de consumidores o la protesta, del mismo modo que la participación en comités expertos internacionales, tienen como resultado incidir en los contornos de los problemas iniciales, cuestionar las tradicionales evaluaciones de beneficio/coste, considerar opciones menos centralizadas o de menor escala, haciéndolas más participativas, influir en los procesos de innovación tecnológica e intervenir en el que hacer de las instituciones.

Modo II: Negociar las tecnologías requiere que los medioambientalistas recojan el compromiso necesario para desarrollar y difundir sus tecnologías o ayudar a aquellos actores que intentan desarrollarlas para que se asocien a ellos. Algunos ejemplos lo constituyen las alianzas de Greenpeace con empresas manufacturadoras de frigoríficos libres de Compuestos Clorofluorocarbonados (CFC), empresas eólicas. También lo realizan las asociaciones forestales aliadas con empresas que consideran estándares medioambientalmente más amigables. Asimismo, las asociaciones de tecnología apropiada que diseñan con criterios de cooperación al desarrollo, 
etc.

En estas asociaciones se realizan campañas de difusión, como por ejemplo, la campaña de Greenpeace para promover los productos y procesos verdes o las bases de datos de productos medioambientales. Otras formas de negociación se llevan a cabo en el terreno del mercado, por ejemplo, estableciendo otras tarifas eléctricas o exigiendo la certificación verde. Muchas Organizaciones No Gubernamentales (ONGs) trabajan en la negociación política para apoyar e impulsar iniciativas públicas en las cuales se exijan y fomenten criterios de sostenibilidad ya sea dentro de los programas de transferencia tecnológica o por medio de subsidios para apoyar proyectos de conservación y cuidado medioambiental.

Así por ejemplo, los movimientos de oposición industrial expuestos por Hess trabajan para alcanzar una moratoria que permita finalizar una práctica tecnológica particular. Como respuesta, una empresa puede ignorar a los activistas, asimismo, puede parar una línea de producción, rediseñar el proceso de producción o innovar completamente los procesos, o incluso, en la misma línea la empresa también puede decidir llevar a cabo una combinación de estas decisiones.

En esta línea, los resultados de las investigaciones en torno a los conflictos locales ante proyectos energéticos en España (BAIGORROTEGUI, 2008; GONZÁLEZ E ESTÉVEZ, 2005) han demostrado que el tránsito entre primero, re-enmarcar una tecnología - movilizar para el "sí" o "no"- y, segundo, negociarla - participar en el diseño o contradiseño - es especialmente importante para los movimientos preocupados y afectados por un proyecto tecnológico situado en su localidad. La relevancia de la cercanía en estas situaciones atiza el despertar público ante determinadas prácticas, por ejemplo, las energéticas, aunque la negociación puede no llegar a concretarse nunca y, como de costumbre, es posible que triunfen los mecanismos de dominación burocrática aliados de proyectos tecnológicos determinados. Para responder a esta tradición de dominación sobre lo local, las víctimas necesitan visualizar sus posibilidades en los contextos globalizados, en otras palabras, requieren "despertar" y ser capaces de solicitar y concretar aliados. 
Los aliados requieren ser hábiles en la gestión de conflictos si lo que desean es hacer visible las demandas de los afectados a través de proyectos tecnológicos. Ellos deben facilitar las transformaciones de identidad en contextos con experiencias de imposición particulares. En otros trabajos he desarrollado la propuesta de una agencia denominada Nicho de Diseños Energéticos para Agencias Afectadas (BAIGORROTEGUI, 2008, cap. V) a favor de gestionar la escalada conflictiva. Este Nicho, en el ámbito de la imposición de proyectos energéticos en lo local, estaría compuesto de grupos de expertos especializados en temas energéticos, jurídicos, políticos, socioeconómicos, financieros, además y particularmente, dotados en técnicas de mediación. Estos profesionales trabajarían en el diagnóstico de una intervención tecnológica en situaciones conflictivas, las cuales surgirían por reacción ante la propuesta impuesta de un proyecto energético particular [VII]. Posteriormente, se ocuparían de gestionar sociotécnicamente el conflicto; ya sea modificando el proyecto original, o sustituyéndolo por otro nuevo. La idea es que la inclusión del Nicho sirva para atraer hacia sí a otros aliados y, con ello, otro enmarcado político energético que favorezca a la localidad receptora de una construcción energética no deseada. Expongo que el Nicho actuaría como una especie de Brigada que facilitaría la labor de apagar el fuego de la contienda, multilateralizando la bipolaridad de la confrontación. Así, el Nicho como entidad emergente en los espacios grises de la institucionalidad, es decir, entre grupos resistentes, movimientos sociales, empresas bienintencionadas, agrupaciones profesionales, todos ellos, dispuestos a intervenir a favor de las localidades, estará supeditado a un análisis estratégico detenido en torno a la factibilidad de su intervención [VIII], la cual no puede fagocitar las movilizaciones locales.

En los espacios de las emergencias [IX], se encuentran variados ejemplos en donde los grupos movilizados, de distinto modo, han logrado modificar sus vidas por medio de un trabajo focalizado en la transformación de prácticas tecnológicas que les afecta, algunos sectores son: salud, energía, grandes infraestructuras, agricultura, alimentación, finanzas, entre 
otros. El impulso gubernamental y empresarial en torno a la concreción de productos y procesos innovadores puede transformarse en la posibilidad de formación de redes con aliados por parte de los grupos movilizados, afectados, amenazados, preocupados, etc. El tránsito hacia la negociación tecnológica por parte de los movilizados requiere de una red socio-técnica aliada, la cual los asesore y proponga vías aledañas a las demandas, preocupaciones e iniciativas de las propias comunidades afectadas.

Dicho esto, expondré y responderé algunas críticas surgidas alrededor de los dilemas intrínsecos referidos a estas propuestas de modalidades alternativas de diseños y proyectos tecnológicos en contextos globales: i) la imposición de un régimen de proyectos, ii) la individualización de la responsabilidad, iii) la cooptación voluntaria de las agrupaciones primero afectadas y luego, beneficiadas y, iv) la actitud y predisposición de los equipos técnicos.

i) La imposición de un régimen de proyectos. Un régimen de proyectos implica la definición de un espacio temporal hacia donde proyectar una intervención; un sistema de mecanismos para evaluar y controlar las actividades; una entidad ante la cual hay que rendir cuentas, la cual por lo general está representada por entidades supranacionales preocupadas por un control financiero poco flexible. La dinámica de proyecto es el lenguaje que separa a las comunidades de expertos de aquellas legas. Las comunidades con otras concepciones de la temporalidad y la paciencia para cumplir y rendir cuentas en sus propios términos suelen, por lo general, quedar subyugadas a la dinámica de proyectos. Como respuesta a esta situación propongo sugerir la distinción entre proyectos para comunidades desfavorecidas del Sur y las comunidades afectadas en localidades occidentales del norte. En ambas existe dominación, pero la posibilidad de flexibilizar los proyectos en son de una apropiación social de los proyectos es distinta. Muchos desafíos tendrá el equipo de diseño de proyectos tecnológicos, el cual proponga indicadores relacionales, es decir, indicadores que conecten la propia práctica de diseño con los resultados esperados por la comunidad beneficiada. Los espacios de interacción 
simbólica son lenguajes que poco a poco han ido integrando proyectos de financiación, sumándose a estas torres de babel de lenguajes en conflicto.

ii) La individualización de la responsabilidad. La valoración de los intereses propios restaría poder a la idea de interés común, propia de la democracia representativa; mientras que, asimismo, asume aproblemáticamente la situación de inclusión con una automática correlación con la responsabilidad civil. Para salir de este embrollo sugiero el diseño de mecanismos de responsabilidad, los cuales la resguarden, tanto por las vías parlamentarias tradicionales, especialmente implantadas en los gobiernos locales, como en el ámbito privado de proyectos por medio de detenidas evaluaciones. Ello, para evitar el lado negativo de la emergencia de redes locales comunitarias y organizaciones voluntaristas, el cual está relacionado con la mayor fragmentación, el menor control y la exclusión riesgosa (KJÆER, 2006, p. 201).

iii) La co-optación voluntaria de las agrupaciones. Este término expuesto por Todt hace referencia a la disminución de la capacidad crítica de las personas que, con posterioridad a un proceso de participación y/o deliberación socio-técnica, hayan resultado favorecidas (TODT, 2003, p. 122). En este entendido, los ciudadanos se cegarían ante los posibles efectos no deseados de una tecnología que han decidido previamente apoyar. Este proceso, al mismo tiempo, provocaría cierta alineación de intereses y objetivos, como describe Kjær: "Miembros de la red podrían desarrollar intereses comunes y activar objetivos, que también los promoverán a ellos mismos. Esto, a su vez, podría no estar de acuerdo con los intereses individuales de los electores" (KJÆER, 2006, p. 15. Traducción mía.). Como esta propuesta la cobijo bajo un tipo de participación en donde los demandantes cuestionan su práctica tecnológica situada y trabajan sobre ella; esto supone que los posibles efectos no deseados serían más visibles y vividos cercanamente y, por tanto, seguirían siendo conflictivos para la localidad. Con esto, determinadas cuestiones a-críticas perceptivas 
de la localidad podrían matizarse cuando el proyecto tecnológico lograse materializarse "comunicativamente" con la localidad (ROWE y FREWER, 2005).

iv) La actitud y predisposición de equipos expertos formados en una cultura científico-técnica predominantemente acrítica son reticentes a trabajar en grupos de personas intranquilas y poco precisas en la definición de los problemas. Si este fuera el caso, se trataría de la confluencia de cosmovisiones de constructores, los cuales parten de premisas incompatibles en torno a la tecnología $[\mathrm{X}]$. Esto podría transformar la propuesta en irrealizable. Aun cuando, los curricula académicos de ingenieros y técnicos dan prueba de las falencias en aspectos de participación social (GÓMEZ et al., 2008), otro panorama se presenta si se observa el crecimiento de ONGs, fundaciones, redes internacionales de científicos e ingenieros concienciados en temas relacionados con la responsabilidad social, el armamentismo y la cooperación internacional [XI].

Asimismo, las características competitivas de los mercados tecnológicos han obligado a ingenieros y tecnólogos a desarrollar capacidades de gestión empresarial al estilo edisoneano. Esto ha hecho que la satisfacción del cliente, políticos, etc. sea una meta que los obliga a negociar muchos más factores que los de factibilidad técnico-económica. En este sentido, los constructores de movilización contenciosa, al proyectar las expectativas de la movilización, coincidirían con los anteriores en el momento de la ampliación del marco de la movilización (SNOW y BENFORD, 2000), pudiendo así incorporar en ella, especialmente en el enmarcado de injusticia, una iniciativa tecnológica asociada en la contienda.

\section{Conclusiones}

En este trabajo propuse vías para que prácticas tecnológicas movilizadas puedan contribuir a los objetivos contrahegemónicos globales. En esta 
propuesta supuse a la tecnología entendida pluralmente, como prácticas, como modos de vida, cercanamente imbricadas en el uso y la cotidianeidad de las personas.

Consecutivamente, las emergencias en los modos de interconexión global me permitieron rescatar que las condiciones que engendran el vacío de legitimación y el dominio translegal pueden ser oportunamente aprovechadas por los movimientos reivindicativos transnacionales en términos recursivos, materiales, visibles. En tercer término, dentro de las estrategias propuestas para estos grupos, la sola escenificación o dramaturgia de los riesgos no resultó suficiente, es el aterrizaje en prácticas específicas, deliberaciones, negociaciones y contraproyectos determinados donde las demandas pueden dejar de estar en manos de una elite exclusiva, debido, entre otros, a que no resulta plausible para las autoridades sostener sus propuestas en base a argumentos deterministas tecnológicos. Lo contrahegemónico aquí resultó determinante, siempre y cuando exista la voluntad de derrocar lo considerado como dado, de sentido común. Esta decisión requiere, en este caso, que las personas despierten de su "sonambulismo tecnológico" y decidan acudir a una "política del reconocimiento desde abajo". Con esta experiencia reflexiva se posibilita que las víctimas logren redefinirse y con ello, se propongan redefinir las reglas del metajuego global. Ahora bien, esta redefinición no provendría exclusivamente de una crítica intensa en contra de la hegemonía, sino también se alimentaría de los logros de llevar a cabo iniciativas contrahegemónicas. Es por esto que renové la propuesta de construir redes sociotécnicas aliadas a los grupos movilizados y propuse hacer de las prácticas tecnológicas contrahegemónicas experiencias concretas de subversión.

\section{Notas Finales}

[1] Este trabajo ha sido posible gracias a la financiación del Programa FPI 2007/2008 del Departamento de Educación, Universidades e Investigación del Gobierno Vasco.

[II] Winner describe el sonambulismo tecnológico según su propia experiencia: "Fue esta tendencia de la comunidad californiana a aceptar transformaciones radicales en la forma de 
vida, a aceptar cambios completos generados en fuentes lejanas casi sin discusión, deliberación o reflexión sobre las consecuencias las que ahora considero realmente sorprendente. Éramos sonámbulos tecnológicos vagando por un constante sueño" (WINNER, 1987, p. 192).

[III] Beck en este punto considera que el capital de legitimación es inconvertible al capital económico, debido a que la fortaleza del capital de legitimación descansa en la confianza pública de los mercados, la que a su vez no se transa en el mercado.

[IV] Existen trabajos que comprueban cómo los cambios en la percepción pública y los patrones de compra, rápidamente se transforman en nuevos sectores de mercado, nuevos estratos de clientes, nuevos productos, etc. (SUMMERTON, 2004).

[V] Las cuales, para Appadurai, constituyen formas de "democracia profunda".

[VI] Por ejemplo, los movimientos orientados a problemáticas particulares. Por ejemplo, aquí se encuentran los movimientos a cargo de la alimentación y agricultura, es decir se trata de agrupaciones críticas acerca del uso de pesticidas, los alimentos genéticamente modificados, las fábricas agrícolas y a favor de la comida orgánica, la agricultura como una forma aledaña para lograr salud y disminuir los riesgos medioambientales asociados a la agricultura industrial. Otro grupo corresponde a los movimientos en torno a la energía, opuestos a las tecnologías nucleares y a las de origen fósil y a favor de las tecnologías de fuentes renovables, el ahorro energético como vías para reducir los riesgos para la salud y los gases de efecto invernadero; los movimientos en torno a la basura y la manufactura promueven la regulación de empresas contaminantes, promoviendo así una orientación hacia los procesos. Estos movimientos son partícipes de la reutilización y el reciclaje, la conservación y recuperación de medios naturales, por tanto, rechazan las incineradoras de gestión de basuras en gran escala, y demandan la gestión de residuos con control local; por su parte, los movimientos en torno a infraestructuras reclaman una planificación del territorio menos centralista, asimismo, demandan diseños urbanistas más participativos, medioambientalmente orientados en contra la creación de anillos de miseria y suburbios urbanos marginados, entre otros. Para más detalles en torno a este tipo de movilizaciones agrupadas temáticamente en Estados Unidos ver Hess, 2007.

[VII] Principalmente proyectos de generación de gran escala (HUGHES, 1987, 1996; SUMMERTON, 1992).

[VIII] La concepción en torno al "Nicho" de este trabajo es más cercana a la de "arena tecnológica" (JØRGENSEN y STRUNGE, 2002) y a la de "objeto conflictivo" (HESS, 2007, HESS y NIEUSMA, 2007) que a la de "nicho" o "nexo" (DOSI, 1982; NELSON y WINTER, 1982; RIP, 2002). La diferencia estriba en las posibilidades de transformación más contingentes y problemáticas social e institucionalmente.

[IX] En el sentido de la sociología de las emergencias que describen Santos y Rodríguez (2007).

[X] Especialmente entre los constructores de artefactos maquiavélicos (LATOUR, 1992) y los constructores de acciones contenciosas (SNOW y BENFORD, 2000).

[XI] Con esto me refiero a movimientos sociales a favor de las tecnologías alternativas (WINNER, 1987), y el desarrollo humano (SCHUMACHER, 1972; CAMACHO, 1989). Los ingenieros y técnicos partícipes en estas movilizaciones se han mostrado cada vez más críticos con su actividad profesional y han decidido crear espacios de reflexión y debate sobre la tecnología y la responsabilidad social de su quehacer (MITCHAM, 1994). Asociaciones como: International Network of Engineers and Scientists for Global Responsability; Ingenieros Sin Fronteras, Engineers against poverty, etc. dan prueba de ello.

\section{Referencias}

APPADURAI, Arjun. Deep Democracy: Urban Governmentality and the Horizon of Politics. Public Culture, Nueva York, v. 14, p. 21-24, 2002. 
BAIGORROTEGUI, Gloria. Dinámica y Gestión de las Electroenergías. EI caso de Boroa. 2008. Tesis (Doctorado) - Universidad del País Vasco/Euskal Herriko Unibersitatea, 2008.

BARNES, Barry; BLOOR, David; HENRY, John. Scientific Knowledge. A sociological Analysis. Londres: Athlone, 1996.

BECK, Ulrich. Poder y Contrapoder en la era global. Barcelona: Paidós, s.d.

BLOOR, David. Knowledge and Social Imagery. Londres: Routledge y Kegan Paul, 1976.

BOURDIEU, Pierre, WACQUANT, Loïc. Una invitación a la Sociología Reflexiva. Coyoacan: Siglo XXI, 2005.

BUNGE, Mario. Filosofía y Tecnología. Monterrey: Universidad Autónoma de Nuevo Leon, 1976.

CALLON, Michel. El Proceso de Construcción de la Sociedad. El estudio de la Tecnología como Herramienta para el Análisis Sociológico. In: DOMĖNECH, M.; TIRADO, Francisco (Comp.). Sociología Simétrica. Barcelona: Gedisa, 1998. p. 143-170.

CAMACHO, Luis. On Technology and Values. In: McLEAN, George; PEGARARO, Olinto (Ed.). The Social Context and Values. Perspectives of the Americas. Londres: Janhan, University Press of America/Council for Research, 1989. p. 125-139.

COLLINS, Harry. Stages in the Empirical Programme of Relativism. Social Studies of Science, Nueva York, v. 1, p. 3-10, 1981.

DOSI, Giovanni. Technological paradigms and technological trajectories: a suggested interpretation of the determinants and directions of technical change, Research policy, v. 11, n. 3, p. 147-62, 1982.

DUSSEL, Enrique. Ética de la Liberación en la edad de la Globalización y la Exclusión. Madrid: Editorial Trotta, 1998.

GIDDENS, Anthony; BECK, Ulrich; LASH, Scott. Modernización Reflexiva: Política, tradición y estética en el orden social moderno. Madrid: Alianza, 1997.

GÓMEZ, Francisco; DURLAN, Cristina; CÁCERES, Santiago; MENDIZÁBAL, Guillermo. La Participación Pública en el Contexto de Proyectos Tecnológicos. Revista CTS, Madrid, v. 10, n. 4, p. 139-157, 2008.

GONZÁLEZ, Marta I.; ESTÉVEZ, Betty. Participación, Comunicación y 
Negociación en Conflictos Ambientales: Energía Eólica marina en el Mar de Trafalgar. Arbor, n. 715, p. 377-92, 2005.

HESS, David. Alternative pathways in science and Industry. Activism, innovation, and the Environment in an era of Globalization. Cambridge: MIT Press, 2007.

NIEUSMA, Dean. What is a Clean Bus? Object conflicts in the greening of urban transit. Sustainability: Science, Practice, \& Policy, v. 3, n. 1, p. 45-58. Disponible en: <http://ejournal.nbii.org/archives/vol3iss1/0608027.hess.html> Acceso el: 12 Enero 2010.

HUGHES, Thomas P. El Impulso Tecnológico. MARX, Leo; SMITH, Marrit Roe (Ed.). Historia y Determinismo Tecnológico. Madrid: Alianza, 1996. p. 117-130.

.The evolution of large technological systems. BIJKER, Wiebe; HUGHES, Thomas P.; PINCH, Trevor (Ed.). The Social Construction of Technological Systems. New directions in the Sociology and History of Technology. Cambridge: MIT Press, 1987. p. 51-82.

Networks of Power. London: Johns Hopkins University Press, 1983. JøRGENSEN, Ulrik; STRUNGE, Lars. Restructuring the Power Arena in Denmark: Shaping Markets, Technology, and Environmental Priorities. In: SØRENSEN, Knut y WILLIAMS, Robin (Ed.). Shaping Technology, Guiding Policy. Massachusetts: Edward Elgar, 2002. p. 293-324.

KAPP, Ernst. Grundlinien einer Philosophie der Technik, Zur Enstehungsgeschite der Kultur als neuen. Dusseldorf, Stern-Verlag Jansen \& Co., 1978.

KJ/ER, Anne. Governance. Cambridge: Polity Press, 2006.

LATOUR, Bruno. Ciencia en Acción. Cómo seguir a los científicos e ingenieros a través de la sociedad. Barcelona: Editorial Labor, 1992.

LAW, John. The anatomy of a socio-technical struggle: The design of the TSR2". In: BIJKER, Wiebe; HUGHES, Thomas; PINCH, Trevor (Ed.). The Social Construction of Technological Systems. New Directions in the Sociology and History of Technoloy. Cambridge: MIT Press, 1987a. p. 44-69.

Technology and heterogeneous engineering: the case of portuguese expansión: In: BIJKER, Wiebe; HUGHES, Thomas; PINCH, Trevor (Ed.). The Social Construction of Technological Systems. New Directions in the Sociology and History of Technology. Cambridge: MIT Press, 1987b. p.111-134.

MARCUSE, Herbert. One dimensional man: studies in the ideology of advanced industrial society. Boston: Beacon, 1964. 
MITCHAM, Carl. Thinking through technology. The Path between engineering and philosophy. Chicago: University of Chicago Press, 1994.

NELSON, Richard; WINTER, Sidney. An evolutionary theory of economic change. Cambridge: Belknap Press of Harvard University Press, 1982.

PINCH, Trevor; BIJKER, Wiebe. The social construction of facts and artefacts: or how the sociology of science and the sociology of technology might benefit Each Other. Social Studies of Science, Londres, v. 14, p. 399-441, 1984.

RIP, Arie. Co-evolution of science, technology and society. REVISIÓN EXPERTA DEL BUNDESMINISTERIUM BILDUNG. Disponible en: <http://www.sciencepolicystudies.de/dok/expertise-rip.pdf> Acceso el: 15 sept. 2008.

ROWE, Gene; FREWER, Lynn. A Typology of Public Engagement Mechanisms. Science, Technology and Human Values, Londres, v. 30, n. 2, p. 251-290, 2005.

SANTOS, Boaventura de Sousa; RODRÍGUEZ, César. EI Derecho y la Globalización desde Abajo, Hacia una legalidad cosmopolita. Barcelona: Anthropos, 2007.

SCHUMACHER, Ernest. F. Small is Beautiful. Madrid: Hermann Blume, 1972.

SMITH, Andrew. The Environment Movement and its Enthusiasm and Resistance to Technology. CONGRESO INTERNACIONAL TECHNOLOGY: BETWEEN ENTHUSIASM AND RESISTANCE, 10-11 May 2005, University of Jyväskylä, Finlandia. Disponible <http://www.jyu.fi/yhtfil/teer2005/abstract/smith.pdf> Acceso el: 22 feb. 2007.

SNOW, David; BENFORD, Robert. Framing processes and social movements: an overview and assessment. Annual Rev. Sociology, Palo Alto, v. 26, p. 611-39, 2000.

SUMMERTON, Jane. Do electrons have politics? Constructing user identities in swedish electricity. Science, Technology \& Human Values, Londres, v. 29, n. 4, p. 486-511, 2004.

District Heating Comes to Town. The Social Shaping of an Energy System. Linköping: Department of Technology and Social Change, 1992.

TAYLOR, Charles. El Multiculturalismo y "la Política del Reconocimiento". México, FCE 1993. 
TODT, Oliver. Potencialidades y Riesgos de la Participación. In: LÓPEZ CEREZO, José Antonio (Ed.). La Democratización de la Ciencia. Donostia: Colección Poliedro, 2003. p. 101-126.

URE, Andrew. Philosophy of Manufacturers or, an exposition of scientific, moral, and commercial economy of the factory system of Great Britain. Londres: Charles Knight, Ludgate-Street, 1835.

WINNER, Langdon. La ballena y el reactor. Una búsqueda de los límites en la era de la alta tecnología. Barcelona: Gedisa, 1987. 